\title{
MCFIS IN HIERARCHICAL BIPREDICTVE PICTURES-BASED VIDEO CODING FOR REFERENCING THE STABLE AREA IN A SCENE
}

\author{
Manoranjan Paul \\ School of Computing and Mathematics, \\ Charles Sturt University, Australia \\ Email:mpaul@csu.edu.au
}

\begin{abstract}
H.264/AVC video coding standard with hierarchical bipredictive picture (HBP) generally outperforms the other prediction structures such as 'IPPP...' and 'IBBP...' through better exploitation of data correlation using the preceding and succeeding reference frames. However, due to the different coding order of frames, the HBP scheme could not fully exploit the data correlations using multiple reference frames for occluded background, repetitive motion, etc. In this paper, we propose a new HBP scheme which uses the most common reference frame in scene (McFIS) as a third reference frame with other two closest bipredictive reference frames assuming that foreground and background areas of the current frame are referenced from the two bipredicted frames and the McFIS respectively. The experimental results confirm that the proposed scheme outperforms two state-of-art algorithms by improving significant image quality with comparable computational time.
\end{abstract}

Index Terms - McFIS, Long term reference frames, uncovered background, hierarchical B-picture, Dual frames, and MRFs.

\section{INTRODUCTION}

H.264/AVC [1][2] with variable block partitioning for motion estimation (ME) and motion compensation (MC) using multiple reference frames (MRFs) technique facilitates better predictions than using one reference frame, for video with features such as repetitive motion, uncovered/occluded background, non-integer pixel displacement, lighting change, etc. The number of reference frames in practical application is limited due to the requirement of codes to identify the reference frame, computational time in ME, and memory buffer size to store decoded frames in both encoder and decoder. Thus, typically the number of reference frames varies from 1 to 5 . If the reference frames in MRFs does not cover those features properly, we will not get any coding improvement and a lot of computation time is wasted.

A number of techniques including [3][4] reduce MRFs associated computational time by avoiding unnecessary searching for various reference frames based on some assumptions on ME, homogeneity, segmentation, etc. Sometimes their assumptions fail due to the diversified video contents, and thus, no time saving is possible. Other techniques [5][6] reduce computational time using dual reference frames (DRFs), one for long term reference (LTR) frame and another for short term reference (STR) frame. In this technique, when $n$th frame is encoding, frame $n-1$ is used as STR

This work is supported by the SINGAPORE MINISTRY OF EDUCATION Academic Research Fund Tier 2 (T208B1218).

\author{
Weisi Lin, Chiew Tong Lau, and Bu-sung Lee \\ School of Computer Engineering, Nanyang \\ Technological University, Singapore. \\ Email: \{wslin, asctlau, ebslee\}@ntu.edu.sg
}

frame and frame $n-N$ (where $N>1$ ) frame is used as LTR frame for $N$ number of frames. The LTR frame then jumps forward by $N$ frames and again remains the same for encoding the next $N$ frames.

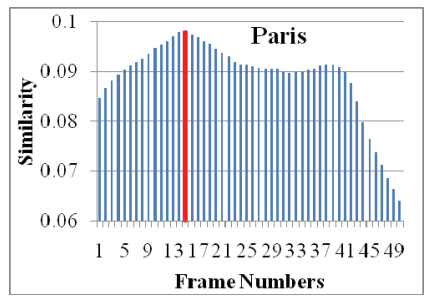

(a)

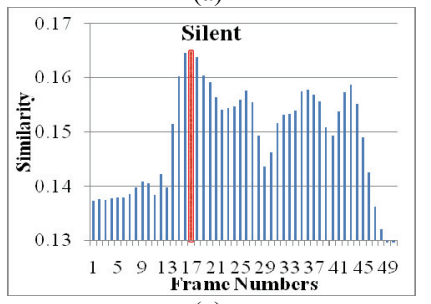

(c)

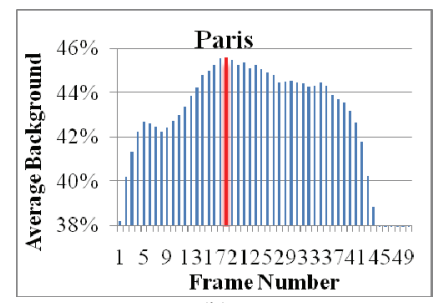

(b)

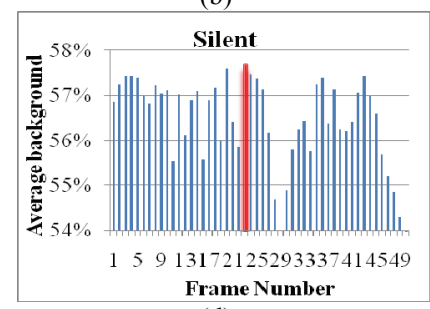

(d)

Fig 1: Average similarity and percentages of backgrounds among frames in a video: (a) \& (c) similarity (expressed in terms of inverse of mean absolute differences (MAD)) among frames respectively, and (b) \& (d) percentages of background among frames of two standard video sequences.

The basic assumption of the DRFs is that the STR frame is found to be useful for local motion i.e., moving areas and the LTR frame is useful for static regions i.e., background areas of the image. Thus, it would be an implicit background (from LTR) and foreground (from STR) referencing technique. Based on the above assumption, our proposition is that the good LTR frame would be the frame which has maximum correlation (i.e., similarity) and/or maximum background areas with the other frames. Fig 1 shows the average correlations and percentages of background for a frame with the rest of the frames in a video. We use first 50 frames of two standard video sequences such as Paris and Silent. We express similarity in terms of inverse of mean absolute difference (MAD) between co-located pixels of two frames. We define a pixel as background pixel if the pixel intensity difference between two colocated pixels is ' 0 ' or ' 1 '. Frame 15 and Frame 17 provide the best similarity (based on the smallest MAD) with other frames of the Paris and Silent video sequences respectively (see Fig 1(a) and (c)). On the other hand, Frame 19 and Frame 23 provide maximum background with other frames of two sequences respectively (see Fig 1(b) and (d)). Those frames are identified by the bold red markers. From the figure, it is clear that no particular frame exhibits better correlation compared to the wide range of frames. Thus, there is no solid ground for an adaptive and/or high quality 
methods [5][6] of the LTR frame selection to be effective for significant coding improvement as they select a LTR frame among the almost equally-probable frames.

A ground truth background of a scene in a video can be better choice to be LTR frame compared to any frame to conform to the implicit background/foreground referencing. Background replenishment and updating techniques [7][8] exploit block-based $\mathrm{ME}$ to generate a background frame for coding purpose. Due to the dependency on block-based motion vectors and lack of adaptability in multi-modal backgrounds for dynamic environment, the background frame generation techniques couldn't perform well. Uncovered background can also be efficiently encoded using sprite/multiple-sprite [9] coding through object segmentation. Most of the video coding applications could not tolerate inaccurate video/object segmentations and expensive computational complexity incurred by segmentation algorithms. Dynamic background modelling (DBM) [10]-[12] using Gaussian Mixture Model is introduced for robust object detection from so called dynamic environment where ground-truth background is impossible due to the practical reasons such as a busy train station, airport, etc. Moreover, static background model does not remain valid due to illumination variations over the time, (un)intentional camera displacement, shadow/reflection of foreground objects, and intrinsic background motions (e.g. waving tree leaves etc). In this paper we modify the latest DBM technique [12] focusing on compression rather than only object detection to get the background frame for LTRF. The background frame is called the most common frame of scene (McFIS).

The flexibility of coding and display order of frames in the H.264 with various decoding delays is exploited by the hierarchical bipredictive picture (HBP) [14][15]. In general, the H.264 with HBP prediction structure outperforms the other prediction structures such as 'IPPP...', 'IBBP...', 'IBPBP...' due to its better exploitation of forward and backward data correlation through preceding and succeeding frame referencing. To maintain bipredicitve referencing, the HBP gains more from the STRF but due to the different coding and display order of frames, it could not take the full advantage of the MRFs benefits (note that LTRF is a sub set of MRFs technique). In this paper, we proposed a new triple MC prediction structure using the two bidirectional reference frames and the McFIS as a uni-directional third reference frame. The experimental results confirm that the proposed scheme outperforms the HBP and the HBP using LTR frame as a third unidirectional reference frame.

The contributions of the paper are (i) the application of the McFIS in the HBP scheme to improve its performance for the static/uncovered areas where traditional frames could not be used efficiently for referencing, and (ii) the application of the McFIS as a third reference frame using unidirectional motion estimation and compensation (i.e., single-hypothesis motion compensation). The rest of the paper is organized as follows: Section 2 will describe the dyadic HBP scheme with three reference frames and Section 3 will discuss the proposed HBP scheme; Section 4 analyses the experimental results and Section 5 concludes the paper.

\section{THE HBP PREDICTION STRUCTURE}

Fig 2 (a) shows a popular dyadic HBP prediction structure with encoding image types, coding, and display order of a GOP (comprises 16 frames) where two bidirectional reference frames (solid arrows only) are used. To get the better coding performance of the HBP structure compared to the other structure (e.g., IPP or IBBP) different quantization parameters (QPs) are used for different hierarchy levels. Normally, finer quantization is applied for the frames which are more frequently used as reference frames for the others frames directly or indirectly. For example, Frame 9 (according to display order) in Fig 2 (a) is used more frequently (4 times directly for frames $5,7,11, \& 13$ and 10 times indirectly for frames $2,3,4,6,8,10,12,14,15$, \& 16) compared to any other Bframes as a reference frame.

The MRFs
echnique can be
applied on the HBP. Intuitively, we make a triple frame referencing technique (HBP-3Ref) under the dyadic HBP structure which is shown in Fig 2 (a) based on the closeness and the availability of the reference frames for a frame. To encode a frame, two solid arrows come from two frames and one dotted arrow comes from the third reference frame. For example, to encode Frame 5, Frame 1 and Frame 9 are used as two bidirectional frames and Frame 17 is

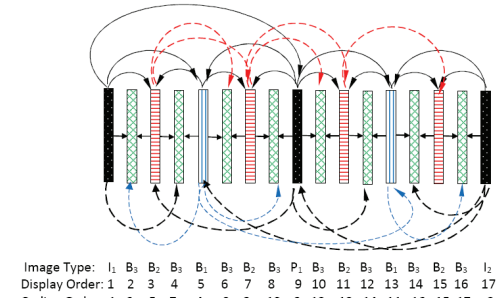

(a)

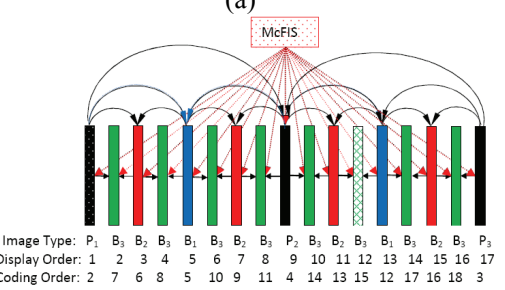

(b)
Fig 2: (a) Dyadic hierarchical B-picture prediction structure using two frames and three frames including the third frame (dotted arrows); and (b) proposed triple frame referencing with the McFIS as the third frame.

used as the third reference frame. On the other hand, to encode Frame 2, Frame 1 and Frame 3 are used as bidirectional frames and Frame 5 is used as the third reference frames. Both examples demonstrate that the MRFs technique in the HBP is not uniform in terms of the distance from the encoding frame and the reference frames. Thus, sometimes it is difficult to exploit the advantages of the MRFs features from the close vicinity frames. Moreover, it could not ensure the implicit background/foreground referencing and thus, it has no physical meaning for referencing.

\section{TRIPLE REFERENCE FRAMES IN THE HBP}

To overcome the limitation of effectiveness using the MRFs technique in the dyadic HBP structure (see Fig 2 (a)) and to exploit the implicit background/foreground referencing, we proposed a new HBP scheme using the McFIS as a third reference frame. By this we assume that motion areas of the current frame would be referenced from the two B-frames and static/uncovered background areas would be referenced from the McFIS.

The diagram of the proposed triple frame MC scheme is shown in Fig 2(b). In this scheme first we generate (generation process will be described in the next sub-section) a McFIS from all frames of the first GOP (in this example 16 frames) of a video/scene. Then we encode it as an inter-frame. All of the frames of a scene are encoded as either B-frame or P-frame with extra reference frame (i.e., McFIS) unless a scene change occurs. If there is scene change we will generate a McFIS again and encode it as Iframe and all of the frames of that scene will be encoded either as B- or P- frames. We note that in the proposed scheme the first and $17^{\text {th }}$ frames (according to the display order) are encoded as Pframes using the McFIS whereas in the existing scheme (see Fig 2(a)), those two frames are encoded as I-frames. In the proposed 
scheme we have used the third frame (i.e., McFIS) as the reference frame for $\mathrm{ME} \& \mathrm{MC}$ for a block in unidirectional way. For example, for a block, firstly we find bits and distortion using two reference frames (marked as solid lines in Fig 2 (b)) by bidirectional ME\&MC (two-hypothesis MC prediction i.e., linear combination of two MC prediction signals); secondly we find bits and distortions using the third frame (i.e., McFIS) for unidirectional by ME\&MC (single-hypothesis MC prediction), and then finally we select one (from the two- hypothesis and the singlehypothesis) based on the Lagrangian multiplier..

To ensure the better coding performance we use finer quantization compared to the quantization of the $\mathrm{P}$-frame. We have used $\mathrm{QP}_{0^{-}} 6$ for the I-frame where $\mathrm{QP}_{0}$ is the $\mathrm{QP}$ of $\mathrm{P}$-frame. We also maintain the same quantization variation mentioned in [14] for different hierarchy levels. For scene change detection we use a simplified algorithm. We calculate sum of absolute difference (SAD) between the McFIS and the current frame. We assume that scene change occurs at the $\mathrm{n} t \mathrm{t}$ frame if the ratio of the SADs at the $n$th frame and the $(n-1)$ th frame is higher than 1.7 . We experimentally observe that this process is computationally efficient and effective compared to the other techniques because the McFIS has the history of the scene [13].

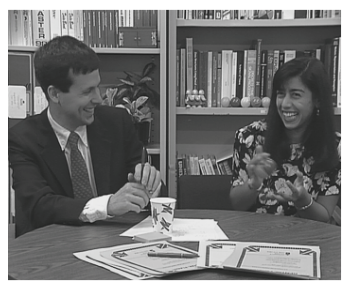

(a)

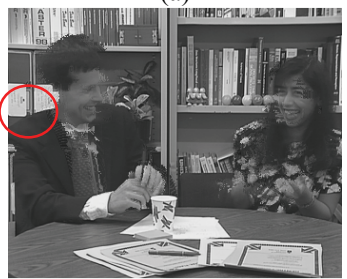

(c)

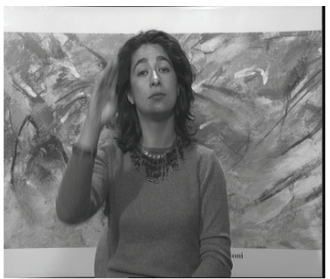

(b)

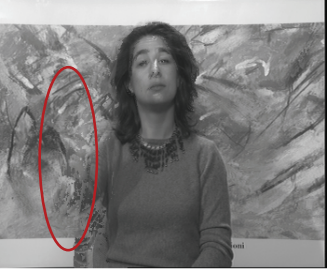

(d)
Fig 3: Examples of McFISes and uncovered/previously occluded background (inside circles) using Paris and Silent video sequences, (a) \& (b) original $16^{\text {th }}$ frame of Paris and Silent sequences respectively; (c) \& (d) corresponding McFISes using first 16 frames of the videos respectively.

\subsection{McFIS generation}

We assume that $k$-th Gaussian at time $t$ representing a pixel intensity with mean $\mu_{k}^{t}$, standard deviation (STD) $\sigma_{k}^{t}$, recent value $\gamma_{k}^{t}$, and weight $\omega_{k}^{t}$ such that $\sum \omega_{k}^{t}=1$. The learning parameter $\alpha$ is used to balance the contribution between the current and past values of parameters such as weight, STD, mean, etc. After initialization, for every new observation $X_{t}$ (pixel intensity at time $t$ ) is first matched against the existing models in order to find one (e.g., $k$ th model) such that $\left|X^{t}-\mu_{k}^{t-1}\right| \leq 2.5 \sigma_{k}^{t-1}$. If such a model exists, update corresponding recent value parameter $\gamma_{k}^{t}$ with $X^{t}$. Other parameters are updated with learning rate as [11]: $\mu_{k}^{t}=(1-\alpha) \mu_{k}^{t-1}+\alpha X^{t} \sigma_{k}^{t^{2}}=(1-\alpha) \sigma_{k}^{t-1^{2}}+\alpha\left(X^{t}-\mu_{k}^{t}\right)^{T}\left(X^{t}-\mu_{k}^{t}\right)$ ; $\omega_{k}^{t}=(1-\alpha) \omega_{k}^{t-1}+\alpha$, and the weights of the remaining Gaussians (i.e., $l$ where $l \neq k$ ) are updated as $\omega_{l}^{t}=(1-\alpha) \omega_{l}^{t-1}$. Afterward, the weights are renormalized. If such a model does not exist, a new Gaussian model is introduced with $\gamma=\mu=X^{t}, \sigma=30$, and $\omega=0.001$ by evicting the $K$-th (based on $w / \sigma$ in descending order) model if it exists. For detailed procedure, please refer [10]-[12].

To get the background pixel intensity from the above mentioned DBM technique for a particular pixel, we take average of the mean pixel value and recent pixel value of the model that has the highest value of weight/standard deviation [13]. In this way we can make a background frame (comprising background pixels) as the McFIS. Two examples of McFIS are shown in Fig 3 using first 16 original frames of Paris and Silent video sequences respectively. Fig 3 (a) \& (b) show the $16^{\text {th }}$ frames of corresponding videos and (c) \& (d) show McFISes. The circles in (c) \& (d) indicate the uncovered/occluded background captured by the corresponding McFIS. To capture the uncovered background by any single frame is quite impossible unless this uncovered background is visible for one frame and that frame is used as LTR frame in the relevant existing approaches. A McFIS is more suitable as a LTR frame than any single pre-encoded frame.

\subsection{McFIS vs. LTR frame as the third reference frame}

Obviously, a frame with its close vicinity has good correlations. The correlation diminishes with the distance. Thus, any frame of a video sequence could not be a good LTR frame. To verify the quality of the McFIS to be a LTR frame, we have compared the percentages of background of other frames with respect to the McFIS and the first frame as the first frame is used more often as a reference frame for other frames. The results are shown in Fig 4 (similar curves are also shown in Fig 1). The figure shows that the first frame exhibits better correlation and more background with respect to first few frames (less than 10 frames) and then worse compared to the McFIS. This figure justifies that the McFIS would be the better choice to be LTR frame due to the more consistence with the distance and more coverage of background (static/uncovered background).
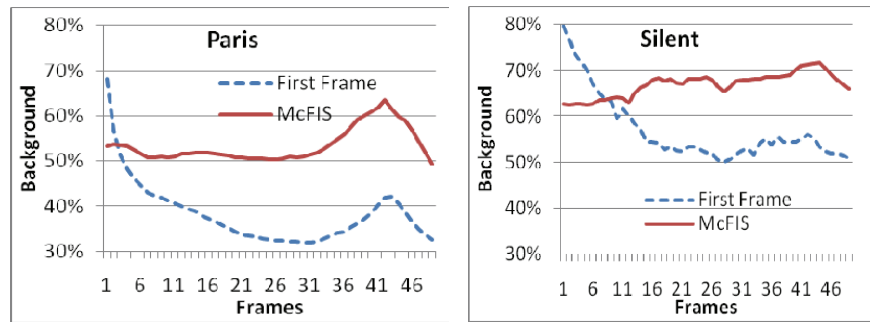

Fig 4: Percentages of backgrounds of the 50 frames against the first frame and the McFIS using two video sequences respectively.

\section{EXPERIMENTAL RESULTS}

To compare the performance of the proposed HBP scheme using McFIS as a third reference frame, we have also implemented the original HBP scheme, the HBP scheme with an extra reference frame (HBP-3Ref), and the HBP scheme with LTR frame (HBPLTR). All the algorithms are implemented based on the H.264 recommendations with $25 \mathrm{~Hz}, \pm 15$ as the search length with quarter-pel accuracy, as 16 as GOP size. The proposed technique has three reference frames and the original HBP technique has two reference frames. Thus, for fair comparison we have selected HBP3-Ref scheme. We also like to see the effectiveness of the McFIS over the LTR frame and the first frame of a scene as a third reference frame in the HBP scheme. To verify this we have selected the HBP-LTR (20 jumping parameter) and HBP- 
FirstFrame (i.e., LTR frame is set to the first frame of a scene) schemes. We have used same QP for high quality LTR frame and the McFIS for fair comparison. We use first 288 frames for videos except the high resolution (4CIF) Popple video (with 112 frames).

Obviously the proposed technique will take some extra operations to generate and encode McFIS compared to the conventional HBP scheme with three reference frames. As we generate and encode the McFIS once for a scene using first few frames, the impact of extra operations is negligible (only $1 \%$ more compared to the H.264-3Ref scheme as shown in Fig 5). Fig 5 shows $16 \sim 50 \%$ and $3 \sim 28 \%$ increase of skip MBs using the proposed and the HBP-LTR schemes compared to the HBP-3Ref scheme as the McFIS represents the stable part of a scene (i.e., background). This figure indicates that the more reduction of bit rates by the proposed technique compared to the others.
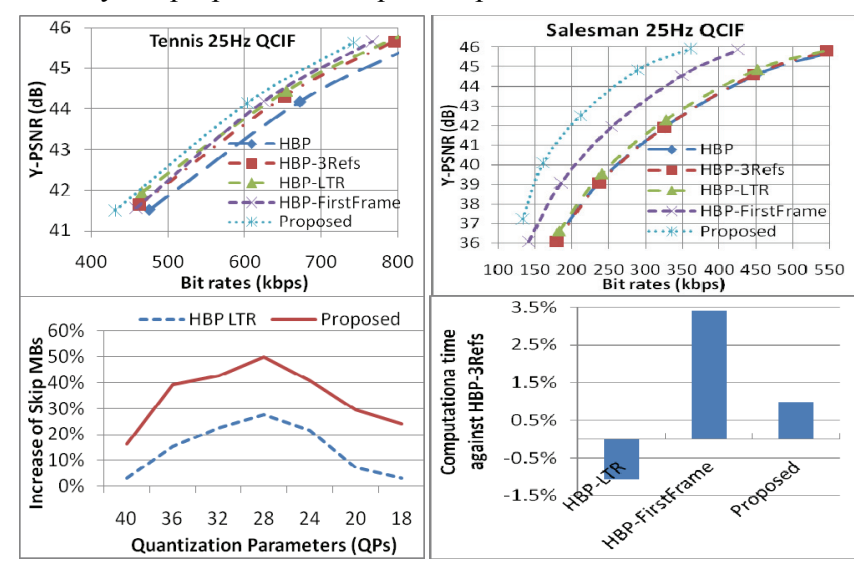

Fig 5: Result comparison in terms of rate-distortion performance (above row) using all relevant algorithms, increase of skip macroblocks (MBs), and computational time requirement by the HBP-LTR, HBP-FirstFrame, the proposed schemes compared to the HBP-3Ref scheme.

Table 1 and Table 2 show a summary of the rate-distortion performance in terms of BD-PSNR and BD-Bit rate for a wide range of bit rates against the HBP structure. Tables reveal that the proposed technique outperforms the HBP-LTR scheme by $1.50 \mathrm{~dB}$ and $15 \%$ respectively on average while 8 standard video sequences are used. Fig 5 (upper row) also shows rate-distortion performance using two standard video sequences. The proposed scheme with its current state could not outperform the state-of-the-art scheme (HBP-3Ref) for the video sequences (e.g., Tempete) with camera motions. It is due to the fact that the proposed technique is not explicitly designed for camera motions.

TABLE 1: PERFORMANCE COMPARISON USING BD-PSNR

\begin{tabular}{cccccc}
\hline \hline Videos & Format & 3Ref & LTR & First Frame & Proposed \\
News & QCIF & 0.02 & 0.45 & 1.99 & 3.29 \\
Hall Objects & QCIF & 0.04 & 0.13 & 1.60 & 2.95 \\
Salesman & QCIF & 0.00 & 0.24 & 2.30 & 4.03 \\
Tennis & QCIF & 0.28 & 0.54 & 0.16 & 1.05 \\
Trevor & QCIF & 0.17 & 0.24 & 0.12 & 0.39 \\
Silent & CIF & 0.12 & 0.36 & 1.93 & 3.07 \\
Paris & CIF & 0.16 & 0.27 & 1.04 & 1.40 \\
Popple & 4CIF & 0.30 & 0.21 & 0.43 & 0.68 \\
Tempete & CIF & 0.71 & 1.34 & 1.11 & 0.68 \\
\hline Average & & $\mathbf{0 . 2 0}$ & $\mathbf{0 . 4 2}$ & $\mathbf{1 . 1 9}$ & $\mathbf{1 . 9 4}$ \\
\hline \hline
\end{tabular}

\section{CONCLUSIONS}

In this paper, we proposed a new hierarchical bipredictive picture based video coding technique using dynamic background frame (named as the McFIS- most common frame of scene) as the third reference frame to overcome multi-frame referencing problem of the existing technique. The proposed technique also outperforms the conventional long term reference frame techniques by better exploiting static and uncovered background through implicit background/foreground referencing. The experimental results show that on average the proposed technique improves the PSNR by $1.50 \mathrm{~dB}$ or compresses the video more by $15 \%$ when compared to the relevant state-of-the-art methods for a wide range of video sequences with comparable computational time.

TABle 2: Performance COMPARISON USING BD-Bit RATE (\%)

\begin{tabular}{lcrrrr}
\hline \hline Video & Format & 3Ref & LTR & First Frame & Proposed \\
News & QCIF & 0.23 & 4.17 & 15.45 & 24.76 \\
Hall Objects & QCIF & 1.79 & 2.68 & 20.93 & 41.32 \\
Salesman & QCIF & 0.22 & 2.44 & 19.99 & 31.06 \\
Tennis & QCIF & 3.95 & 6.73 & 4.76 & 12.86 \\
Trevor & QCIF & 2.34 & 2.92 & 2.47 & 5.12 \\
Silent & CIF & 1.84 & 4.98 & 21.81 & 32.99 \\
Paris & CIF & 1.94 & 2.51 & 12.51 & 16.78 \\
Popple & 4CIF & 4.43 & 3.21 & 6.76 & 10.16 \\
Tempete & CIF & 9.09 & 16.2 & 12.92 & 8.23 \\
\hline Average & & $\mathbf{2 . 8 7}$ & $\mathbf{5 . 0 9}$ & $\mathbf{1 3 . 0 7}$ & $\mathbf{2 0 . 3 6}$ \\
\hline \hline
\end{tabular}

\section{REFERENCES}

[1] T. Wiegand, G. J. Sullivan, G. Bjøntegaard, and A. Luthra, "Overview of the H.264/AVC Video Coding Standard," IEEE Trans. on Circuits and Systems for Video Technology (CSVT), 13(7), 560-576, 2003.

[2] M. Paul and M. Murshed, "Video Coding focusing on block partitioning and occlusions," IEEE Trans. on Image Processing, vol. 19, no. 3, pp. 691-701, 2010.

[3] T. -Y. Kuo, H. -J. Lu, "Efficient Reference Frame Selector for H.264," IEEE Trans. On CSVT, vol. 18, no. 3, pp. 400-405, 2008,

[4] K. Hachicha, D. Faura, O. Romain, and P. Garda, "Accelerating the multiple reference frames compensation in the H.264 video coder," Journal of Real-Time Image Processing, Springer, vol. 4, no. 1, pp. 55-65, 2009.

[5] M. Tiwari and P. C. Cosman, "Selection of Long-Term Reference Frames in Dual-Frame Video Coding Using Simulated Annealing," IEEE Signal Processing Letter, vol. 15, pp. 249-252, 2008.

[6] D. Liu, D. Zhao, X. Ji, and W. Gao, "Dual Frame Motion Compensation With Optimal Long-Term Reference Frame Selection and Bit Allocation," IEEE Transactions on CSVT, 20(3), pp. 325 - 339, 2010.

[7] D. Hepper, "Efficiency analysis and application of uncovered background prediction in a low bit rate image coder," IEEE Transaction on Communication, vol. 38, no. 9, pp. 1578-1584, 1990.

[8] P. Wilkins, J. Bankoski, and Y. Xu, "System and method for video encoding using constructed reference frame," patent: 20100061461, 2010.

[9] A. Krutz, A. Glantz, and T. Sikora, "Background Modelling for Video Coding: From Sprites to Global Motion Temporal Filtering," IEEE Int. Symposium on Circuits and Systems, pp. 2179-2182, 2010.

[10] C. Stauffer and W. E. L. Grimson, "Adaptive background mixture models for real-time tracking," IEEE CVPR, vol. 2, pp. 246-252, 1999.

[11] D.-S. Lee, "Effective Gaussian mixture learning for video background subtraction," IEEE Transactions on PAMI, 27(5), pp. 827-832, May 2005.

[12] M. Haque, M. Murshed, and M. Paul, "Improved Gaussian mixtures for robust object detection by adaptive multi-background generation," IEEE Int. Conference on Pattern Recognition, pp. 1-4, 2008.

[13] M. Paul, W. Lin, C. T. Lau, and B. -S. Lee, "Video coding using the most common frame in scene," IEEE ICASSP, pp. 734-737, 2010.

[14] H. Schwarz, D. Marpe, and T. Wiegand, "Analysis of hierarchical Bpictures and MCTF," IEEE ICME, pp. 1929-1932, 2006.

[15] M. Flierl and B. Girod, "Generalized B Pictures and the Draft H.264/AVC Video Compression Standard," IEEE Trans. on CSVT, 13(7), $587-597,2003$. 